\title{
Formononetin inhibits lipopolysaccharide-induced release of high mobility group box 1 by upregulating SIRT1 in a PPARס- dependent manner
}

\author{
Jung Seok Hwang ${ }^{1}$, Eun Sil Kang ${ }^{1}$, Sung Gu Han ${ }^{1}{ }^{\text {, }}$ Dae-Seog Lim ${ }^{2}$, Kyung Shin Paek ${ }^{3}$, Chi-Ho Lee ${ }^{1}$, Han \\ Geuk Seo ${ }^{\text {Corresp. } 1}$ \\ ${ }^{1}$ Department of Food Science and Biotechnology of Animal Products, Sanghuh College of Life Sciences, Konkuk University, Seoul, Korea \\ 2 Department of Biotechnology, CHA University, Seongnam, Korea \\ 3 Department of Nursing, Semyung University, Jechon, Korea \\ Corresponding Author: Han Geuk Seo \\ Email address: hgseo@konkuk.ac.kr
}

Background. The release of high mobility group box 1 (HMGB1) induced by inflammatory signals acts as a cellular alarmin to trigger a chain of inflammatory responses. Although the inflammatory actions of HMGB1 are well studied, less is known about the therapeutic agents that can impede its release. This study investigated whether the isoflavonoid formononetin can modulate HMGB1 release in cellular inflammatory responses.

Methods. RAW264.7 murine macrophages were exposed to lipopolysaccharide (LPS) in the presence or absence of formononetin. The levels of HMGB1 release, sirtuin 1 (SIRT1) expression, and HMGB1 acetylation were analyzed by immunoblotting and real-time polymerase chain reaction. The effects of resveratrol and sirtinol, an activator and inhibitor of SIRT1, respectively, on LPS-induced HMGB1 release were also evaluated.

Results. Formononetin modulated cellular inflammatory responses by suppressing the release of HMGB1 by macrophages exposed to LPS. In RAW264.7 cells, formononetin significantly attenuated LPS-induced release of HMGB1 into the extracellular environment, which was accompanied by a reduction in its translocation from the nucleus to the cytoplasm. In addition, formononetin significantly induced mRNA and protein expression of SIRT1 in a peroxisome proliferator-activated receptor $\delta$ (PPARס)-dependent manner. These effects of formononetin were dramatically attenuated in cells treated with small interfering RNA (siRNA) against PPARס or with GSK0660, a specific inhibitor of PPARס, indicating that PPARס is involved in formononetin-mediated SIRT1 expression. In line with these effects, formononetinmediated inhibition of HMGB1 release in LPS-treated cells was reversed by treatment with SIRT1targeting siRNA or sirtinol, a SIRT1 inhibitor. By contrast, resveratrol, a SIRT1 activator, further potentiated the inhibitory effect of formononetin on LPS-induced HMGB1 release, revealing a possible mechanism by which formononetin regulates HMGB1 release through SIRT1. Furthermore, modulation of SIRT1 expression by transfection of SIRT1- or PPARס-targeting siRNA significantly counteracted the inhibitory effects of formononetin on LPS-induced HMGB1 acetylation, which was responsible for HMGB1 release.

Discussion. This study shows for the first time that formononetin inhibits HMGB1 release by decreasing HMGB1 acetylation via upregulating SIRT1 in a PPAR 8 -dependent manner. Formononetin consequently exhibits anti-inflammatory activity. Identification of agents, such as formononetin, which can block HMGB1 release, may help to treat inflammation-related disorders. 
3 Jung Seok Hwang ${ }^{1}$, Eun Sil Kang ${ }^{1}$, Sung Gu Han ${ }^{1}$, Dae-Seog Lim², Kyung Shin Paek ${ }^{3}$, Chi-Ho

4 Lee $^{1}$, and Han Geuk Seo ${ }^{1}$

$5{ }^{1}$ Department of Food Science and Biotechnology of Animal Products, Sanghuh College of Life

6 Sciences, Konkuk University, 120 Neungdong-ro, Gwangjin-gu, Seoul 05029, Korea

$7{ }^{2}$ Department of Biotechnology, CHA University, 335 Pangyo-ro, Bundang-gu, Seongnam 13488,

8 Korea

$9 \quad{ }^{3}$ Department of Nursing, Semyung University, 65 Semyung-ro, Jechon 27136, Korea

10 Correspondence to:

11 Han Geuk Seo

12 Department of Food Science and Biotechnology of Animal Products, Sanghuh College of Life

13 Sciences, Konkuk University, 120 Neungdong-ro, Gwangjin-Gu, Seoul 05029, Korea

14 Tel.: +82 2450 0428, Fax: +8224551044

15 E-mail: hgseo@konkuk.ac.kr 


\section{ABSTRACT}

Background. The release of high mobility group box 1 (HMGB1) induced by inflammatory signals acts as a cellular alarmin to trigger a chain of inflammatory responses. Although the inflammatory actions of HMGB1 are well studied, less is known about the therapeutic agents that can impede its release. This study investigated whether the isoflavonoid formononetin can modulate HMGB1 release in cellular inflammatory responses.

Methods. RAW264.7 murine macrophages were exposed to lipopolysaccharide (LPS) in the presence or absence of formononetin. The levels of HMGB1 release, sirtuin 1 (SIRT1) expression, and HMGB1 acetylation were analyzed by immunoblotting and real-time polymerase chain reaction. The effects of resveratrol and sirtinol, an activator and inhibitor of SIRT1, respectively, on LPS-induced HMGB1 release were also evaluated.

Results. Formononetin modulated cellular inflammatory responses by suppressing the release of HMGB1 by macrophages exposed to LPS. In RAW264.7 cells, formononetin significantly attenuated LPS-induced release of HMGB1 into the extracellular environment, which was accompanied by a reduction in its translocation from the nucleus to the cytoplasm. In addition, formononetin significantly induced mRNA and protein expression of SIRT1 in a peroxisome proliferator-activated receptor $\delta(\mathrm{PPAR} \delta)$-dependent manner. These effects of formononetin were dramatically attenuated in cells treated with small interfering RNA (siRNA) against PPAR $\delta$ or with GSK0660, a specific inhibitor of PPAR $\delta$, indicating that PPAR $\delta$ is involved in formononetin-mediated SIRT1 expression. In line with these effects, formononetin-mediated inhibition of HMGB1 release in LPS-treated cells was reversed by treatment with SIRT1targeting siRNA or sirtinol, a SIRT1 inhibitor. By contrast, resveratrol, a SIRT1 activator, further potentiated the inhibitory effect of formononetin on LPS-induced HMGB1 release, revealing a possible mechanism by which formononetin regulates HMGB1 release through SIRT1. 
40 Furthermore, modulation of SIRT1 expression by transfection of SIRT1- or PPAR $\delta$-targeting 41 siRNA significantly counteracted the inhibitory effects of formononetin on LPS-induced HMGB1 42 acetylation, which was responsible for HMGB1 release.

43 Discussion. This study shows for the first time that formononetin inhibits HMGB1 release by 44 decreasing HMGB1 acetylation via upregulating SIRT1 in a PPAR $\delta$-dependent manner. 45 Formononetin consequently exhibits anti-inflammatory activity. Identification of agents, such as 46 formononetin, which can block HMGB1 release, may help to treat inflammation-related 47 disorders. 


\section{INTRODUCTION}

High mobility group box 1 (HMGB1), a non-histone DNA-binding protein, is a well-conserved nuclear protein that has multiple functions depending on its cellular location. In the nucleus, HMGB1 plays roles in DNA replication, transcription, recombination, and maintenance of chromosome stability (Stros, 2010). However, when released by stressed cells, HMGB1 plays a critical role in the inflammatory response and is a late proinflammatory marker in many diseases including sepsis (Andersson \& Harris, 2010; Abdulahad et al., 2010; Sims et al., 2010; Stros, 2010; Zhang et al., 2009). Recent reports show that post-translational modifications of HMGB1, such as acetylation and phosphorylation, are associated with its translocation and release in inflammatory cells exposed to pathogen-related molecules including lipopolysaccharide (LPS) (Bonaldi et al., 2003; Ito et al., 2007; Youn \& Shin, 2006). The importance of extracellular HMGB1 in the inflammatory response has been demonstrated in inflammatory conditions; a neutralizing anti-HMGB1 antibody and HMGB1 antagonists attenuate cellular damage induced by inflammation (Wang et al., 1999; Davé et al., 2009). These reports indicate the importance of pathways or molecules that regulate HMGB1 release from activated inflammatory cells.

Sirtuin 1 (SIRT1) is a type III histone deacetylase that controls multiple genetic programs by acting on histone and non-histone substrates (Xie et al., 2013). This protein is a vital regulator of various physiological and metabolic processes such as energy metabolism (Purushotham et al., 2009), aging (Tissenbaum \& Guarente, 2001), apoptosis (Motta et al., 2004), mitochondrial biogenesis (Brenmoehl \& Hoeflich, 2013), and the stress response (Brunet et al., 2004). Recent studies also demonstrate that SIRT1 is directly involved in cellular inflammatory responses by deacetylating inflammation-related transcription factors such as nuclear factor-kappa B (NF-KB) and activator protein-1 (AP-1), which suppresses the transcription of diverse inflammationresponsive genes (Feige \& Auwerx, 2008; Zhang \& Kraus, 2010). Furthermore, we demonstrated 
72 that transcriptional upregulation of SIRT1 by peroxisome proliferator-activated receptor $\delta$ 73 (PPAR $\delta$ ) and PPAR $\gamma$ inhibits HMGB1 release by decreasing its LPS-induced acetylation, 74 indicating that SIRT1 deacetylates HMGB1 (Hwang et al., 2012; Hwang et al., 2014). While genetic ablation of SIRT1 increases the secretion and expression of proinflammatory cytokines, SIRT1 activators prevent the production of tumor necrosis factor- $\alpha$, monocyte chemoattractant protein-1, and interleukin (IL)-8 (Dong et al., 2014; Yang et al., 2007), highlighting the central role of SIRT1 in the regulation of cellular inflammatory responses.

Formononetin, a herbal isoflavonoid, was isolated from the medicinal plant Astragalus membranaceus and has a variety of biological activities including anti-tumor (Auyeung et al., 2012; Chen et al., 2011), wound healing (Huh et al., 2011), antioxidant (Mu et al., 2009), and anti-inflammatory (Krenn \& Paper, 2009; Lai et al., 2013) effects. Specifically, formononetin inhibits inflammation-related gene expression by blocking the NF- $\mathrm{KB}$ and AP-1 signaling pathways in animal models of inflammatory diseases (Chen et al., 2007; Hämäläinen et al., 2007). In particular, synthetic derivatives of formononetin increase the activity of PPAR $\delta$, indicating this compound is useful to treat inflammation-related diseases (Zhao et al., 2017). Furthermore, we showed that activation of PPAR $\delta$ and PPAR $\gamma$ by specific ligands induces SIRT1 expression in human coronary artery endothelial cells (Kim et al., 2012) and RAW264.7 cells (Hwang et al., 2014). Thus, we hypothesized that formononetin may modulate cellular inflammatory responses by inhibiting HMGB1 release via upregulation of SIRT1. Here, we show that formononetin reduces LPS-induced HMGB1 acetylation by upregulating SIRT1 in a PPAR $\delta$ dependent manner, thereby blocking HMGB1 release into the extracellular environment. 


\section{MATERIALS \& METHODS}

\section{Materials}

95 Formononetin, actinomycin D (Act D), cycloheximide (CHX), Ponceau S solution, resveratrol, 96 sirtinol, MTT, LPS (Escherichia coli 0111:B4), curcumin, genistein, and an anti- $\beta$-actin 97 polyclonal antibody were obtained from Sigma-Aldrich Co. (St. Louis, MO, USA). GSK0660 98 and the luciferase assay system were purchased from Tocris Bioscience (Bristol, UK) and 99 Promega (Madison, WI, USA), respectively. Monoclonal antibodies specific for HMGB1 and 100 PPAR $\delta$ were supplied by Epitomics (Burlingame, CA, USA). Monoclonal antibodies specific for 101 acetyl-lysine, lamin B, and $\alpha$-tubulin as well as a polyclonal antibody specific for SIRT1 were 102 supplied by Santa Cruz Biotechnology (Dallas, TX, USA).

\section{Cell culture}

Human primary peripheral blood macrophages and RAW264.7 murine macrophage-like cells were purchased from STEMCELL Technologies (Vancouver, BC, Canada) and the Korean Cell Line Bank (Seoul, Korea), respectively. Primary human macrophages and RAW264.7 cells were maintained in RPMI (Roswell Park Memorial Institute) 1640 and DMEM (Dulbecco's modified Eagle medium) containing antibiotics and $10 \% \mathrm{FCS}$ at $37^{\circ} \mathrm{C}$ in a $5 \%$ humidified $\mathrm{CO}_{2}$ incubator, respectively.

\section{Cell viability assays}

RAW264.7 cells were stimulated with $30 \mu \mathrm{M}$ formononetin for the indicated duration or the indicated dose of formononetin for $24 \mathrm{~h}$ in 24-well plates. Thereafter, MTT assays and trypan blue exclusion were performed to determine the cell viability. For trypan blue exclusion, the collected cells were mixed with trypan blue solution $(0.4 \%)$, and then viable cells were 
115 determined by a hemocytometer. For MTT assay, the cells were incubated for final $2 \mathrm{~h}$ in medium

116 containing MTT solution $(0.1 \mathrm{mg} / \mathrm{ml})$. Following removing the medium, the absorbance at 570

$117 \mathrm{~nm}$ was measured using formazan crystals solution dissolved in acidified isopropanol.

118 Western blot analysis

119 Protein levels were analyzed by immunoblot as described previously (Hwang et al., 2015).

120 Briefly, RAW264.7 cells washed with ice-cold PBS were lysed and aliquots of the resulting 121 whole-cell lysates or conditioned media were analyzed by immunoblot with indicated antibodies.

122 Immuno-reactive bands were detected using WesternBright ECL (Advansta Co., Melon Park, CA, 123 USA).

\section{Measurement of extracellular HMGB1}

Levels of HMGB1 released into culture media were determined using a previously described method (Hwang et al., 2012). Briefly, the relative amounts of HMGB1 were determined in the conditioned media of RAW264.7 cells treated with the indicated reagents for the indicated durations. The $80 \%$ ice-cold acetone was used to precipitate the proteins in the conditioned media. After centrifugation, the pellets were obtained and washed with $80 \%$ ice-cold acetone. Following resuspension in SDS-PAGE sample buffer, the levels of HMGB1 released into culture media were analyzed by immunoblot.

\section{Fractionation of nuclear and cytoplasmic proteins}

Cellular fractions were prepared using a previously described method (Hwang et al., 2015). Briefly, RAW264.7 cells were washed in PBS, suspended in lysis solution for $15 \mathrm{~min}$ at $4^{\circ} \mathrm{C}$ to swell. Nonidet P-40 (final $0.1 \%$ concentration) was added to the lysates and then vortexed vigorously for $20 \mathrm{sec}$. Following centrifugation $(13,000 \times \mathrm{g})$ for $20 \mathrm{sec}$, the supernatant 
137 containing cytosolic fraction was obtained and the resulting pellet was lysed by a PRO-PREP

138 Protein Extraction Solution. Following standing for $20 \mathrm{~min}$ on ice, the nuclear fraction 139 (supernatant) was obtained by centrifugation.

\section{Reporter gene assay}

The luciferase construct containing mouse SIRT1 promoter was a gift from Dr. Toren Finkel (NIH, MD, USA). The promoter activity of SIRT1 was measured as described previously (Hwang et al., 2014). Briefly, $1 \mu \mathrm{g}$ of the SIRT1 luciferase reporter plasmid and $0.5 \mu \mathrm{g}$ of pSV $\beta$ Gal (SV40 $\beta$-galactosidase expression vector) were introduced into RAW264.7 cells by SuperFect reagent (Qiagen, Valencia, CA, USA). After 38 h, the cells were treated with GSK0660 for 30 min prior to stimulation with formononetin for $24 \mathrm{~h}$. Then, the cells were lysed by adding the luciferase reporter lysis buffer (Promega) and then aliquots of the lysates were used to determine luciferase activity.

\section{Small interfering RNA (siRNA)-mediated gene silencing}

The indicated siRNA was introduced into RAW264.7 cells in serum-containing medium using SuperFect (Qiagen) as described previously (Hwang et al., 2014). Briefly, siRNA targeting scrambled non-specific sequences (Ambion, Austin, TX, USA), PPAR $\delta$ (Ambion), or SIRT1 designed against nucleotides (5'-TAATATCTGAGGCACTTCA-3' and 5'TGAAGTGCCTCAGATATTA-3') of mouse (Bioneer, Daejeon, Korea) was introduced into the cells for $6 \mathrm{~h}$. The cells were then cultured for further $38 \mathrm{~h}$ in fresh medium. At which time, the indicated reagents were added into the cells for the indicated durations. Gene silencing was analyzed by immunoblot. 


\section{Real-time polymerase chain reaction (PCR)}

Levels of SIRT1 and HMGB1 mRNA were analyzed by real-time PCR as described previously (Hwang et al., 2014). Briefly, total RNA was converted into cDNA by a reverse transcription kit (TOPscript RT DryMIX, Enzynomics, Seoul, Korea). Real-time PCR was carried out using equal amount of cDNA in a $20 \mu \mathrm{l}$ reaction solution containing primers and $1 \times$ SYBR PCR mix (Takara Bio Inc., Otsu, Japan). The PCR condition: initial denaturation at $94^{\circ} \mathrm{C}$ for $20 \mathrm{~min}$, followed by 42 cycles of $25 \mathrm{~s}$ at $95^{\circ} \mathrm{C}, 44 \mathrm{~s}$ at $58.2^{\circ} \mathrm{C}$, and $40 \mathrm{~s}$ at $72^{\circ} \mathrm{C}$. The primers were as follows: SIRT1, 5'-AGAACCACCAAAGCGGAAA-3' and 5'-TCCCACAGGAGACAGAAACC-3'; HMGB1, 5'-TACCGCCCCAAAATCAAAGG-3' and 5'-TCTCATAGGGCTGCTTGTCA-3'; and GAPDH, 5'-CATGGCCTTCCGTGTTCCTA-3' and 5'-CCTGCTTCACCACCTTCTTGAT-3'.

\section{Co-immunoprecipitation}

Immunoprecipitation was performed using a previously described method (Hwang et al., 2015). Briefly, the protein G Sepharose was added to whole-cell lysates to pre-clear and then the precleared lysates were mixed with $1 \mu \mathrm{g}$ of an anti-HMGB1 antibody. After incubation overnight at $4^{\circ} \mathrm{C}$, the mixture was reacted with protein $\mathrm{G}$ Sepharose for $4 \mathrm{~h}$. Mixtures were extensively washed with PBS and then boiled in gel-loading buffer. The immunoblot analysis was performed using an anti-acetyl-lysine antibody (Santa Cruz Biotechnology).

\section{Statistical analysis}

Data are expressed as means \pm standard error (SE). The significance in statistical analysis was evaluated by a one-way ANOVA, followed by Tukey-Kramer test. A value of $p<0.05$ was considered statistically significant. 
179

180

181

182

\section{RESULTS}

\section{Formononetin inhibits LPS-induced release of HMGB1 in both murine and human} macrophages

To determine the optimal concentration of formononetin, we determined the viability of RAW264.7 cells treated with various concentrations of formononetin for $24 \mathrm{~h}$ or with $30 \mu \mathrm{M}$ formononetin for various durations. Treatment with concentrations of formononetin up to $30 \mu \mathrm{M}$ did not elicit cytotoxic effects on RAW264.7 cells, and cell viability remained high following treatment with $30 \mu \mathrm{M}$ formononetin for up to 5 days (Figure 1A and 1B). Thus, we selected 30 $\mu \mathrm{M}$ formononetin as the optimal concentration for subsequent experiments using RAW264.7 cells.

Because many herbal compounds including curcumin and genistein reported to exhibit antiinflammatory activity (Biswas \& Rahman, 2008; Dharmappa et al., 2010), we compared the effect of formononetin on the HMGB1 release with curcumin and genistein in RAW264.7 cells treated with LPS. The level of HMGB1 released into culture media was increased in RAW264.7 cells exposed to LPS, and this increase was markedly reduced in the presence of herbal compounds. In particular, the effects of formononetin and curcumin were superior to those of genistein (Figure 1C). By contrast, neither LPS nor formononetin affected the expression level of endogenous HMGB1 (Figure 1D). Similar results were obtained from the human primary macrophages, indicating that formononetin affects LPS-induced HMGB1 release, but not HMGB1 expression, in both murine and human macrophages (Figure 1E).

HMGB1 is reported to translocate from the nucleus into the cytoplasm in response to inflammatory signals such as LPS (Bonaldi et al., 2003; Youn \& Shin, 2006). Therefore, we examined whether formononetin affects this translocation of HMGB1 in LPS-stimulated RAW264.7 cells. While translocation of HMGB1 into the cytoplasm was increased in cells 
exposed to LPS, this was significantly suppressed by formononetin (Figure 1F). However, the expression of HMGB1 mRNA was not affected by formononetin in cells treated with or without LPS (Figure 1G). These results suggest that formononetin decreases the release of HMGB1 by inhibiting its translocation rather than expression in LPS-primed RAW264.7 cells.

\section{Formononetin upregulates SIRT1 expression in RAW264.7 cells}

Formononetin increased protein expression of SIRT1 in RAW264.7 cells in a concentration- and time-dependent manner. SIRT1 protein expression was significantly increased in cells treated with 20-30 $\mu \mathrm{M}$ formononetin for $24 \mathrm{~h}$ (Figure $2 \mathrm{~A}$ ) and peaked at $12-24 \mathrm{~h}$ in cells treated with 30 $\mu \mathrm{M}$ formononetin (Figure 2B). Similarly, the mRNA level of SIRT1 was time-dependently upregulated by formononetin (Figure 2C). In addition, the inhibitory effect of formononetin on the LPS-stimulated release of HMGB1 was significant at $6 \mathrm{~h}$ pretreatment and the maximal inhibitory effect of formononetin was observed with a pre-treatment of $24 \mathrm{~h}$ which corresponds to the time of maximal induction of SIRT1 expression upon formononetin treatment (Figure 2D).

To elucidate the mechanisms by which formononetin induces SIRT1 expression, we determined the effects of Act D (a RNA synthesis inhibitor) and CHX (a protein synthesis inhibitor). While formononetin significantly increased mRNA expression of SIRT1, this was significantly reduced in the presence of Act D or CHX (Figure 2E). These results indicate that de novo synthesis of mRNA as well as of proteins that act on the SIRT1 gene promoter is indispensable for the induction of SIRT1 mRNA by formononetin in RAW264.7 cells.

\section{Formononetin induces SIRT1 expression via PPAR $\delta$ in RAW264.7 cells}

To further examine the mechanisms by which formononetin upregulates SIRT1 expression, we evaluated the role of PPAR $\delta$, a nuclear receptor that regulates the transcription of a variety of 
225 target genes (Kidani \& Bensinger, 2012; Mangelsdorf et al., 1995), by transfecting RAW264.7

226 cells with siRNA against PPAR $\delta$. The protein level of PPAR $\delta$ was reduced in cells transfected

227 with PPAR $\delta$-targeting siRNA, but not in cells transfected with control siRNA composed of a pool

228 of nonspecific sequences (Figure 2F). Transfection of PPAR $\delta$-targeting siRNA attenuated the

229 induction of SIRT1 expression by formononetin, whereas transfection of control siRNA did not

230 (Figure 2G). In line with these findings, GSK0660, a specific inhibitor of PPAR $\delta$, significantly

231 attenuated the formononetin-induced increase in SIRT1 promoter activity (Figure 2H). These

232 results suggest that formononetin upregulates SIRT1 expression via PPAR $\delta$ at the transcriptional 233 level.

\section{SIRT1 is essential for inhibition of LPS-induced HMGB1 release by formononetin}

To investigate the direct effect of SIRT1 on LPS-induced HMGB1 release, we examined the levels of SIRT1 protein and released HMGB1 in RAW264.7 cells exposed to LPS in the presence or absence of formononetin. A high level of HMGB1 was released upon LPS treatment, whereas this was reduced in the presence of formononetin. On the other hand, the level of SIRT1 protein was significantly suppressed in LPS-treated RAW264.7 cells. However, this LPS-mediated repression of SIRT1 was recovered in the presence of formononetin, indicating that SIRT1 is critical for modulation of LPS-induced HMGB1 release by formononetin (Figure 3A).

To further clarify the functional significance of formononetin-mediated upregulation of SIRT1 in RAW264.7 cells, we manipulated the expression and activity of SIRT1 using siRNA or chemicals. The levels of SIRT1 protein were diminished in cells transfected with SIRT1 siRNA, however control siRNAs had no effect on the levels of either protein (Figure 3B). Transfection of SIRT1-targeting siRNA significantly attenuated the inhibitory effect of formononetin on LPSinduced HMGB1 release (Figure 3C). Consistently, inhibition of SIRT1 activity by sirtinol also 
248 prevented inhibition of HMGB1 release by formononetin (Figure 3D). By contrast, activation of

249 SIRT1 by resveratrol inhibited LPS-induced HMGB1 release. Furthermore, resveratrol treatment 250 potentiated the inhibitory effects of formononetin, suggesting that SIRT1 plays a role in the 251 suppression of HMGB1 release by formononetin (Figure 3E). These results indicate that 252 formononetin inhibits LPS-induced HMGB1 release by regulating SIRT1 expression.

\section{SIRT1-mediated deacetylation of HMGB1 underlies the inhibition of its release by} formononetin

Inflammatory signal-mediated acetylation of HMGB1 is critical for its release into the extracellular compartment and acetylated HMGB1 is a substrate of SIRT1 (Bonaldi et al., 2003; Hwang et al., 2014; Rickenbacher et al., 2014); therefore, we evaluated whether formononetin affects LPS-induced acetylation of HMGB1. When RAW264.7 cells were stimulated with LPS for $6 \mathrm{~h}$, the level of acetylated HMGB1 in an immunoprecipitate obtained using an anti-HMGB1 antibody was significantly enhanced. However, formononetin reduced this increase in acetylated HMGB1 in a concentration-dependent manner, indicating that formononetin is involved in the deacetylation of HMGB1 primed by LPS (Figure 4A).

To evaluate whether this inhibition of LPS-induced HMGB1 acetylation by formononetin correlates with the level of SIRT1 in RAW264.7 cells, we knocked down SIRT1. Transfection of SIRT1-targeting siRNA significantly prevented the decrease in acetylated HMGB1 by formononetin in LPS-exposed RAW264.7 cells (Figure 4B). Transfection of PPAR $\delta$-targeting siRNA elicited the same effect (Figure 4C). These results indicate that formononetin reduces HMGB1 acetylation via PPAR $\delta$-mediated upregulation of SIRT1, thereby inhibiting the release of HMGB1 into the extracellular milieu. 
HMGB1 plays physiological and pathological roles by acting as an intracellular structural protein and an extracellular cytokine (Ueda \& Yoshida, 2010; Andersson \& Tracey, 2011). Although the roles of extracellular HMGB1 in the pathogenesis of inflammatory disease are well established, the regulatory mechanisms underlying HMGB1 release or therapeutic agents that can impede its release was not fully elucidated. Here, we showed that formononetin inhibited LPS-induced release of HMGB1 in RAW264.7 cells. This inhibition was mediated by PPAR $\delta$-dependent upregulation of SIRT1, a class III deacetylase involved in cellular inflammatory responses (Brunet et al., 2004; Yeung et al., 2004; Zhang et al., 2010). SIRT1 expression was also upregulated at the transcriptional level in RAW264.7 cells treated with formononetin. Modulation of SIRT1 expression and activity by siRNAs and chemicals abolished the inhibitory effect of formononetin on HMGB1 release. In addition, SIRT1 upregulated by formononetin deacetylated HMGB1, which inhibited release of HMGB1. This demonstrates that formononetin has antiinflammatory actions in LPS-stimulated RAW264.7 cells. These results are in line with the previous finding that formononetin elicits anti-inflammatory effects by upregulating PPAR $\gamma$ expression in an animal model of LPS-induced acute lung injury (Ma et al., 2013). In addition, formononetin attenuates hydrogen peroxide- and IL-1 $\beta$-induced activation of NF- $\mathrm{B}$ in retinal ganglion cells and the insulinoma cell line INS-1, respectively (Jia et al., 2014; Wang et al., 2012). Although the molecular mechanisms underlying formononetin-mediated antiinflammatory responses have not been fully elucidated until now, the present study clearly demonstrated that formononetin inhibits LPS-induced release of HMGB1 in the mouse macrophage cell line RAW264.7, suggesting that formononetin is a promising therapeutic agent for inflammation-related disorders.

The release of HMGB1 during inflammatory responses is closely linked with its post- 
294 translational modifications such as acetylation and phosphorylation (Bonaldi et al., 2003; Youn \&

295 Shin, 2006). Consistent with previous studies, formononetin inhibited LPS-induced acetylation of 296 HMGB1, leading to suppression of its release. This effect of formononetin on HMGB1 release was intimately correlated with the level of SIRT1 expression, indicating that SIRT1 deacetylates HMGB1 and thereby inhibits its release. This result is in line with previous reports indicating that SIRT1 deacetylates inflammation-related transcription factors such as AP-1 and NF- $\mathrm{B}$, and thereby modulates the progression of inflammation by suppressing the transcription of diverse inflammation-related genes (Yang et al., 2007; Yeung et al., 2004; Zhang et al., 2010). These results provide a rationale for the use of SIRT1 activators as therapeutic agents in inflammatory diseases as shown previous studies using resveratrol to activate the SIRT1 (Xu et al., 2014; Dong et al., 2015). In fact, a recent study demonstrated that inflammatory diseases are closely associated with a reduced SIRT1 protein level (Xie et al., 2013). Because release of HMGB1 is intimately correlated with its post-translational modifications along with decreased SIRT1 expression, it may be possible to suppress inflammatory reactions by inducing SIRT1 expression using formononetin.

Formononetin-mediated upregulation of SIRT1 was critical for inhibition of LPS-induced HMGB1 release. SIRT1, a $\mathrm{NAD}^{+}$-dependent deacetylase, is implicated in diverse cellular processes, such as stress responses, aging, energy metabolism, and inflammation, through its deacetylase activity (Brunet et al., 2004; Chen et al., 2005; Cohen et al., 2004; Feige \& Auwerx, 2008; Yeung et al., 2004; Zhang \& Kraus, 2010; Zhang et al., 2010). Although transcriptional regulation of SIRT1 in mammalian cells has been mainly established in the context of energy metabolism-related pathways such as caloric restriction (Chen et al., 2005; Cohen et al., 2004), transcription factors, including TLX, BRCA1, HIC1, and E2F1, are also implicated in the regulation of SIRT1 expression (Chen et al., 2005; Iwahara et al., 2009; Wang et al., 2006; Wang et al., 2008). However, the transcriptional regulation of SIRT1 is complex and the underlying 
319 mechanism is unclear. The nuclear hormone receptor PPAR $\delta$ was recently demonstrated to 320 regulate SIRT1 expression in various cell lineages (Kim et al., 2012; Okazaki et al., 2010). 321 PPAR $\delta$ was initially shown to promote SIRT1 expression in human hepatocytes via an 322 unconventional mechanism in which specificity protein 1 plays a central role, rather than the 323 PPAR-response element (Okazaki et al., 2010). PPAR $\delta$ activation also induces SIRT1 expression 324 in vascular endothelial cells (Kim et al., 2012). On the other hand, formononetin, a compound 325 extracted from $S$. flavescens roots, significantly increases PPAR $\delta$ activity in a concentration326 dependent manner (Quang et al., 2013), indicating that transactivation of PPAR $\delta$ by 327 formononetin is linked to SIRT1 expression. This result is in line with our finding that 328 formononetin induced SIRT1 expression in a PPAR $\delta$-dependent manner. 


\section{CONCLUSIONS}

330 To our knowledge, this is the first report to show that formononetin inhibits HMGB1 release by 331 upregulating SIRT1 transcription and thus inducing HMGB1 deacetylation in LPS-treated 332 RAW264.7 cells. This novel finding has important implications for our understanding of the 333 molecular mechanism underlying the transcriptional regulation of SIRT1 as well as the anti334 inflammatory effect of formononetin. In light of these observations, formononetin-mediated 335 enhancement of SIRT1 activity in macrophages is likely a new therapeutic strategy for 336 inflammatory disorders. 


\section{REFERENCES}

Abdulahad DA, Westra J, Limburg PC, Kallenberg CG, Bijl M. 2010. HMGB1 in systemic lupus Erythematosus: Its role in cutaneous lesions development. Autoimmunity Reviews 9:661-665 DOI 10.1016/j.autrev.2010.05.015.

Andersson U, Harris HE. 2010. The role of HMGB1 in the pathogenesis of rheumatic disease. Biochimica et Biophysica Acta 1799:141-148 DOI 10.1016/j.bbagrm.2009.11.003.

Andersson U, Tracey KJ. 2011. HMGB1 is a therapeutic target for sterile inflammation and infection. Annual Review of Immunology 29:139-162 DOI 10.1146/annurev-immunol030409-101323.

Auyeung KK, Law PC, Ko JK. 2012. Novel anti-angiogenic effects of formononetin in human colon cancer cells and tumor xenograft. Oncology Reports 28:2188-2194. DOI 10.3892/or.2012.2056.

Biswas S, Rahman I. 2008. Modulation of steroid activity in chronic inflammation: a novel antiinflammatory role for curcumin. Molecular Nutrition \& Food Research 52:987-994. DOI 10.1002/mnfr.200700259.

Bonaldi T, Talamo F, Scaffidi P, Ferrera D, Porto A, Bachi A, Rubartelli A, Agresti A, Bianchi ME. 2003. Monocytic cells hyperacetylate chromatin protein HMGB1 to redirect it towards secretion. The EMBO Journal 22:5551-5560.

Brenmoehl J, Hoeflich A. 2013. Dual control of mitochondrial biogenesis by sirtuin 1 and sirtuin 3. Mitochondrion 13:755-761 DOI 10.1016/j.mito.2013.04.002.

Brunet A, Sweeney LB, Sturgill JF, Chua KF, Greer PL, Lin Y, Tran H, Ross SE, Mostoslavsky R, Cohen HY, Hu LS, Cheng HL, Jedrychowski MP, Gygi SP, Sinclair DA, Alt FW, Greenberg ME. 2004. Stress-dependent regulation of FOXO transcription factors by the SIRT1 deacetylase. Science 303:2011-2015 DOI 10.1126/science.1094637. 
Chen CY, Peng WH, Tsai KD, Hsu SL. 2007. Luteolin suppresses inflammation-associated gene expression by blocking NF-kappaB and AP-1 activation pathway in mouse alveolar macrophages. Life Sciences 81:1602-1614 DOI 10.1016/j.lfs.2007.09.028.

Chen D, Steele AD, Lindquist S, Guarente L. 2005. Increase in activity during calorie restriction requires Sirt1. Science 310:1641 DOI 10.1126/science.1118357.

Chen J, Zeng J, Xin M, Huang W, Chen X. 2011. Formononetin induces cell cycle arrest of human breast cancer cells via IGF1/PI3K/Akt pathways in vitro and in vivo. Hormone and Metabolic Research 43:681-686 DOI 10.1055/s-0031-1286306.

Chen WY, Wang DH, Yen RC, Luo J, Gu W, Baylin SB. 2005. Tumor suppressor HIC1 directly regulates SIRT1 to modulate p53-dependent DNA-damage responses. Cell 123:437448 DOI 10.1016/j.cell.2005.08.011.

Cohen HY, Miller C, Bitterman KJ, Wall NR, Hekking B, Kessler B, Howitz KT, Gorospe M, de Cabo R, Sinclair DA. 2004. Calorie restriction promotes mammalian cell survival by inducing the SIRT1 deacetylase. Science 305:390-392 DOI 10.1126/science.1099196.

Davé SH, Tilstra JS, Matsuoka K, Li F, DeMarco RA, Beer-Stolz D, Sepulveda AR, Fink MP, Lotze MT, Plevy SE. 2009. Ethyl pyruvate decreases HMGB1 release and ameliorates murine colitis. Journal of Leukocyte Biology 86:633-643. DOI 10.1189/jlb.1008662.

Dharmappa KK, Mohamed R, Shivaprasad HV, Vishwanath BS. 2010. Genistein, a potent inhibitor of secretory phospholipase A2: a new insight in down regulation of inflammation. Inflammopharmacology 18:25-31. DOI 10.1007/s10787-009-0018-8.

Dong WW, Liu YJ, Lv Z, Mao YF, Wang YW, Zhu XY, Jiang L. 2015. Lung endothelial barrier protection by resveratrol involves inhibition of HMGB1 release and HMGB1-induced mitochondrial oxidative damage via an Nrf2-dependent mechanism. Free Radical Biology \& Medicine 88:404-416. DOI 10.1016/j.freeradbiomed.2015.05.004. 
Inhibitory effects of resveratrol on foam cell formation are mediated through monocyte chemotactic protein-1 and lipid metabolism-related proteins. International Journal of Molecular Medicine 33:1161-1168 DOI 10.3892/ijmm.2014.1680.

Feige JN, Auwerx J. 2008. Transcriptional targets of sirtuins in the coordination of mammalian physiology. Current Opinion in Cell Biology 20:303-309. DOI 10.1016/j.ceb.2008.03.012.

Hämäläinen M, Nieminen R, Vuorela P, Heinonen M, Moilanen E. 2007. Anti-inflammatory effects of flavonoids: genistein, kaempferol, quercetin, and daidzein inhibit STAT-1 and NFkappaB activations, whereas flavone, isorhamnetin, naringenin, and pelargonidin inhibit only NF-kappaB activation along with their inhibitory effect on iNOS expression and NO production in activated macrophages. Mediators of Inflammation 2007:45673. DOI $10.1155 / 2007 / 45673$.

Huh JE, Nam DW, Baek YH, Kang JW, Park DS, Choi DY, Lee JD. 2011. Formononetin accelerates wound repair by the regulation of early growth response factor-1 transcription factor through the phosphorylation of the ERK and p38 MAPK pathways. International Immunopharmacology 11:46-54 DOI 10.1016/j.intimp.2010.10.003.

Hwang JS, Choi HS, Ham SA, Yoo T, Lee WJ, Paek KS, Seo HG. 2015. Deacetylationmediated interaction of SIRT1-HMGB1 improves survival in a mouse model of endotoxemia. Scientific Reports 5:15971 DOI 10.1038/srep15971.

Hwang JS, Kang ES, Ham SA, Yoo T, Lee H, Paek KS, Park C, Kim JH, Lim DS, Seo HG. 2012. Activation of peroxisome proliferator-activated receptor $\gamma$ by rosiglitazone inhibits lipopolysaccharide-induced release of high mobility group box 1. Mediators of Inflammation 2012:352807 DOI 10.1155/2012/352807.

Hwang JS, Lee WJ, Kang ES, Ham SA, Yoo T, Paek KS, Lim DS, Do JT, Seo HG. 2014. Ligand-activated peroxisome proliferator-activated receptor- $\delta$ and $-\gamma$ inhibit lipopolysaccharide-primed release of high mobility group box 1 through upregulation of 
SIRT1. Cell Death and Disease 5:e1432 DOI 10.1038/cddis.2014.406.

Ito I, Fukazawa J, Yoshida M. 2007. Post-translational methylation of high mobility group box 1 (HMGB1) causes its cytoplasmic localization in neutrophils. The Journal of Biological Chemistry 282:16336-16344 DOI 10.1074/jbc.M608467200.

Iwahara N, Hisahara S, Hayashi T, Horio Y. 2009. Transcriptional activation of NAD+dependent protein deacetylase SIRT1 by nuclear receptor TLX. Biochemical and Biophysical Research Communications 386:671-675 DOI 10.1016/j.bbrc.2009.06.103.

Jia WC, Liu G, Zhang CD, Zhang SP. 2014. Formononetin attenuates hydrogen peroxide (H2O2)-induced apoptosis and NF-kB activation in RGC-5 cells. European Review for Medical and Pharmacological Sciences 18:2191-2197.

Kidani Y, Bensinger SJ. 2012. Liver X receptor and peroxisome proliferator-activated receptor as integrators of lipid homeostasis and immunity. Immunological Reviews 249:72-83 DOI 10.1111/j.1600-065X.2012.01153.x.

Kim MY, Kang ES, Ham SA, Hwang JS, Yoo TS, Lee H, Paek KS, Park C, Lee HT, Kim JH, Han CW, Seo HG. 2012. The PPAR $\delta$-mediated inhibition of angiotensin II-induced premature senescence in human endothelial cells is SIRT1-dependent. Biochemical Pharmacology 84:1627-1634 DOI 10.1016/j.bcp.2012.09.008.

Krenn L, Paper DH. 2009. Inhibition of angiogenesis and inflammation by an extract of red $\begin{array}{lllll}\text { clover (Trifolium pratense } & \text { L.). Phytomedicine 16:1083-1088 DOI }\end{array}$ 10.1016/j.phymed.2009.05.017.

Lai PK, Chan JY, Cheng L, Lau CP, Han SQ, Leung PC, Fung KP, Lau CB. 2013. Isolation of anti-inflammatory fractions and compounds from the root of Astragalus membranaceus. Phytotherapy Research 27:581-587 DOI 10.1002/ptr.4759.

Ma Z, Ji W, Fu Q, Ma S. 2013. Formononetin inhibited the inflammation of LPS-induced acute lung injury in mice associated with induction of PPAR gamma expression. Inflammation 


\section{6:1560-1566 DOI 10.1007/s10753-013-9700-5.}

Mangelsdorf DJ, Thummel C, Beato M, Herrlich P, Schütz G, Umesono K, Blumberg B, Kastner P, Mark M, Chambon P, Evans RM. 1995. The nuclear receptor superfamily: the second decade. Cell 83:835-839 DOI 10.1016/0092-8674(95)90199-X.

Motta MC, Divecha N, Lemieux M, Kamel C, Chen D, Gu W, Bultsma Y, McBurney M, Guarente L. 2004. Mammalian SIRT1 represses forkhead transcription factors. Cell 116:551-563 DOI 10.1016/S0092-8674(04)00126-6.

Mu H, Bai YH, Wang ST, Zhu ZM, Zhang YW. 2009. Research on antioxidant effects and estrogenic effect of formononetin from Trifolium pratense (red clover). Phytomedicine 16:314-319 DOI 10.1016/j.phymed.2008.07.005.

Okazaki M, Iwasaki Y, Nishiyama M, Taguchi T, Tsugita M, Nakayama S, Kambayashi M, Hashimoto K, Terada Y. 2010. PPARbeta/delta regulates the human SIRT1 gene transcription via Sp1. Endocrine Journal 57:403-413 DOI 10.1507/endocrj.K10E-004.

Purushotham A, Schug TT, Xu Q, Surapureddi S, Guo X, Li X. 2009. Hepatocyte-specific deletion of SIRT1 alters fatty acid metabolism and results in hepatic steatosis and inflammation. Cell Metabolism 9:327-338. DOI 10.1016/j.cmet.2009.02.006.

Quang TH, Ngan NT, Minh CV, Kiem PV, Tai BH, Nhiem NX, Thao NP, Luyen BT, Yang SY, Kim YH. 2013. Anti-inflammatory and PPAR transactivational properties of flavonoids from the roots of Sophora flavescens. Phytotherapy Research 27:1300-1307 DOI $10.1002 /$ ptr.4871.

Rickenbacher A, Jang JH, Limani P, Ungethüm U, Lehmann K, Oberkofler CE, Weber A, Graf R, Humar B, Clavien PA. 2014. Fasting protects liver from ischemic injury through Sirt1-mediated downregulation of circulating HMGB1 in mice. Journal of Hepatology 61:301-308 DOI 10.1016/j.jhep.2014.04.010.

Sims GP, Rowe DC, Rietdijk ST, Herbst R, Coyle AJ. 2010. HMGB1 and RAGE in 
461

inflammation and cancer. Annual Review of Immunology 28:367-388 DOI 10.1146/annurev.immunol.021908.132603.

Stros M. 2010. HMGB proteins: interactions with DNA and chromatin. Biochimica et Biophysica Acta 1799:101-113 DOI 10.1016/j.bbagrm.2009.09.008.

Tissenbaum HA, Guarente L. 2001. Increased dosage of a sir-2 gene extends lifespan in Caenorhabditis elegans. Nature 410:227-230 DOI 10.1038/35065638.

Ueda T, Yoshida M. 2010. HMGB proteins and transcriptional regulation. Biochimica et Biophysica Acta 1799:114-118 DOI 10.1016/j.bbagrm.2009.11.005.

Wang C, Chen L, Hou X, Li Z, Kabra N, Ma Y, Nemoto S, Finkel T, Gu W, Cress WD, Chen J. 2006. Interactions between E2F1 and SirT1 regulate apoptotic response to DNA damage. Nature Cell Biology 8:1025-1031 DOI 10.1038/ncb1468.

Wang H, Bloom O, Zhang M, Vishnubhakat JM, Ombrellino M, Che J, Frazier A, Yang H, Ivanova S, Borovikova L, Manogue KR, Faist E, Abraham E, Andersson J, Andersson U, Molina PE, Abumrad NN, Sama A, Tracey KJ. 1999. HMG-1 as a late mediator of endotoxin lethality in mice. Science 285:248-251 DOI 10.1126/science.285.5425.248.

Wang RH, Zheng Y, Kim HS, Xu X, Cao L, Luhasen T, Lee MH, Xiao C, Vassilopoulos A, Chen W, Gardner K, Man YG, Hung MC, Finkel T, Deng CX. 2008. Interplay among BRCA1, SIRT1, and Survivin during BRCA1-associated tumorigenesis. Molecular Cell 32:11-20 DOI 10.1016/j.molcel.2008.09.011.

Wang Y, Zhu Y, Gao L, Yin H, Xie Z, Wang D, Zhu Z, Han X. 2012. Formononetin attenuates

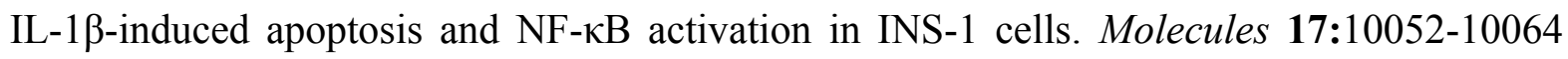
DOI 10.3390/molecules170910052.

Xie J, Zhang X, Zhang L. 2013. Negative regulation of inflammation by SIRT1. Pharmacological Research 67:60-67. DOI 10.1016/j.phrs.2012.10.010. 
486

487

Novel role of resveratrol: suppression of high-mobility group protein box 1 nucleocytoplasmic translocation by the upregulation of sirtuin 1 in sepsis-induced liver injury. Shock 42:440-447. DOI 10.1097/SHK.0000000000000225.

Yang SR, Wright J, Bauter M, Seweryniak K, Kode A, Rahman I. 2007. Sirtuin regulates cigarette smoke-induced proinflammatory mediator release via RelA/p65 NF-kappaB in macrophages in vitro and in rat lungs in vivo: implications for chronic inflammation and aging. American Journal of Physiology-Lung Cellular and Molecular Physiology 292:L567576 DOI 10.1152/ajplung.00308.2006.

Yeung F, Hoberg JE, Ramsey CS, Keller MD, Jones DR, Frye RA, Mayo MW. 2004. Modulation of NF-kappaB-dependent transcription and cell survival by the SIRT1 deacetylase. The EMBO Journal 23:2369-2380 DOI 10.1038/sj.emboj.7600244.

Youn JH, Shin JS. 2006. Nucleocytoplasmic shuttling of HMGB1 is regulated by phosphorylation that redirects it toward secretion. The Journal of Immunology 177:78897897 DOI 10.4049/jimmunol.177.11.7889.

Zhang R, Chen HZ, Liu JJ, Jia YY, Zhang ZQ, Yang RF, Zhang Y, Xu J, Wei YS, Liu DP, Liang CC. 2010. SIRT1 suppresses activator protein-1 transcriptional activity and cyclooxygenase-2 expression in macrophages. The Journal of Biological Chemistry 285:7097-7110 DOI 10.1074/jbc.M109.038604.

Zhang S, Zhong J, Yang P, Gong F, Wang CY. 2009. HMGB1, an innate alarmin, in the pathogenesis of type 1 diabetes. Journal of Clinical and Experimental Pathology 3:24-38.

Zhang T, Kraus WL. 2010. SIRT1-dependent regulation of chromatin and transcription: linking $\mathrm{NAD}(+)$ metabolism and signaling to the control of cellular functions. Biochimica et Biophysica Acta 1804:1666-1675. DOI 10.1016/j.bbapap.2009.10.022.

Zhao MJ, Wang SS, Jiang Y, Wang Y, Shen H, Xu P, Xiang H, Xiao H. 2017. Hypolipidemic effect of XH601 on hamsters of Hyperlipidemia and its potential mechanism. Lipids in 
$511 \quad$ Health and Disease 16:85 DOI 10.1186/s12944-017-0472-z. 


\section{Figure 1}

Effects of formononetin on the LPS-induced release and translocation of HMGB1.

( $A$ and B) RAW264.7 cells cultured in serum-free medium for $16 \mathrm{~h}$ were treated with the indicated concentrations of formononetin for $24 \mathrm{~h}$ (A) or with $30 \mathrm{mM}$ formononetin for the indicated durations (B). Cell viability was determined by the MTT (A) and trypan blue exclusion (B) assays. (C) RAW264.7 cells maintained in serum-free medium for $16 \mathrm{~h}$ were stimulated with LPS in the presence or absence of indicated herbal compound for $24 \mathrm{~h}$. Equal volumes of conditioned media were analyzed by immunoblotting. Ponceau S staining was used as the loading controls. (D and E) RAW264.7 cells (D) or human primary macrophages (E) cultured in serum-free medium for $16 \mathrm{~h}$ were stimulated with LPS in the presence or absence of formononetin for $24 \mathrm{~h}$. Equal volumes of conditioned media or aliquots of wholecell lysates were analyzed by immunoblotting. Ponceau $\mathrm{S}$ staining and $\beta$-actin were used as the loading controls. Black and gray bars indicate secreted HMGB1 and intracellular HMGB1, respectively. (F) RAW264.7 cells treated with LPS in the presence or absence of formononetin for $24 \mathrm{~h}$ were fractionated into nuclear $(\mathrm{N})$ and cytosolic $(\mathrm{C})$ fractions. The localization of HMGB1 was determined by Western blot analysis with the indicated antibodies. (G)

RAW264.7 cells were treated with LPS in the presence or absence of formononetin. Following incubation for $24 \mathrm{~h}$, total RNA was isolated and the levels of SIRT1 mRNA were analyzed by real-time PCR. The results are plotted as the means \pm SE ( $n=3$ or 4 ). ${ }^{*} p<0.05,{ }^{* *} p<0.01$

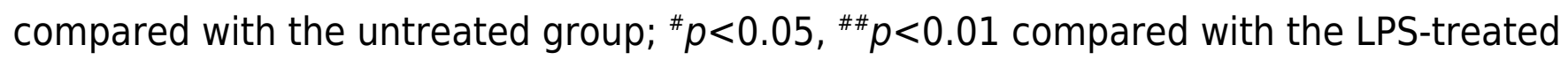
group. 
A

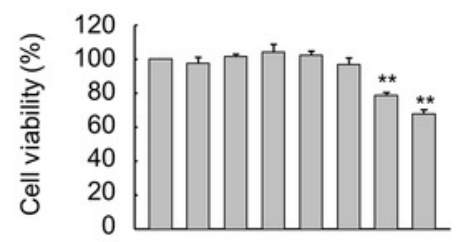

Formononetin $(\mu \mathrm{M}) \quad 0 \quad 1 \quad 5 \quad 1020304050$

Time $(24 \mathrm{~h})$

B

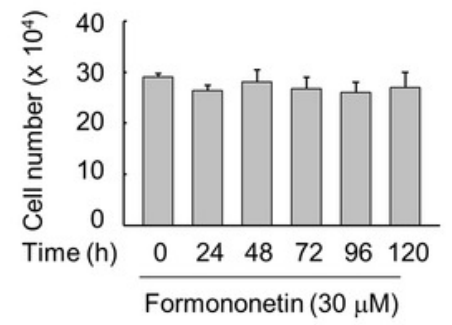

C

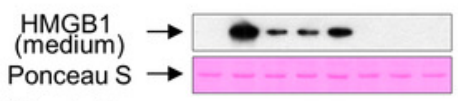

LPS $(100 \mathrm{ng} / \mathrm{ml})$

Formononetin $(8 \mu \mathrm{gg} / \mathrm{ml})-\ldots+\ldots+\ldots$

Curcumin $(8 \mu \mathrm{g} / \mathrm{ml})$

$--+-\cdots+$

Genistein $(8 \mu \mathrm{g} / \mathrm{ml})$
D

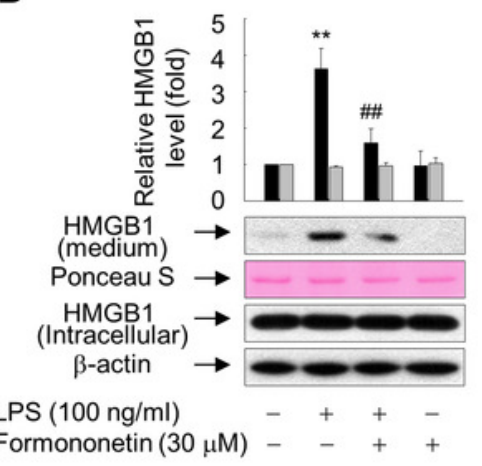

E

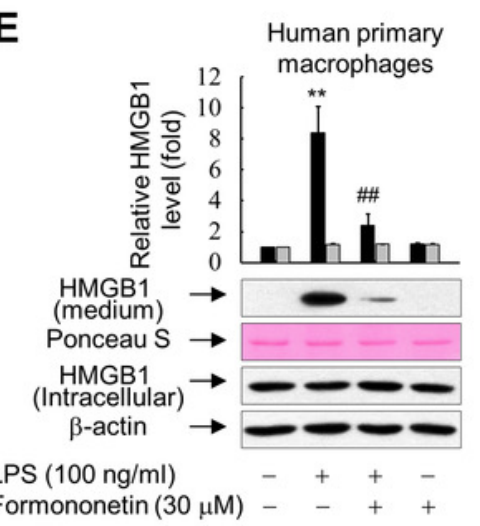

$\mathbf{F}$
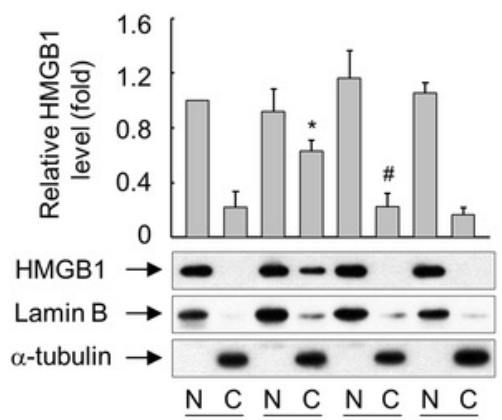

LPS $(100 \mathrm{ng} / \mathrm{ml})$

Formononetin $(30 \mu \mathrm{M}) \quad-\quad-\quad+\quad+$

G

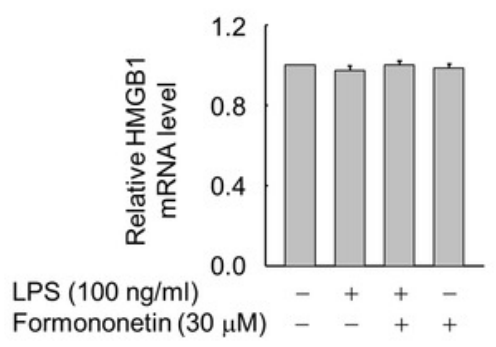




\section{Figure 2}

Involvement of PPARd in formononetin-mediated upregulation of SIRT1 in RAW264.7 cells.

( $A$ and $B$ ) Cells cultured in serum-free medium for $16 \mathrm{~h}$ were incubated with various concentrations of formononetin for $24 \mathrm{~h}$ (A) or with $30 \mathrm{mM}$ formononetin for the indicated durations (B). Aliquots of whole-cell lysates were analyzed by immunoblotting.

Representative blots are provided. Fold changes in the SIRT1/ $\beta$-actin ratio relative to that in the untreated group are shown as means $\pm S E(n=3)$. (C) Cells cultured in serum-free medium for $16 \mathrm{~h}$ were stimulated with formononetin for the indicated durations. After incubation for $24 \mathrm{~h}$, total RNA was isolated and the levels of SIRT1 mRNA were analyzed by real-time PCR. The results are expressed as the means $\pm S E(n=3)$. (D) Cells maintained in serum-free medium for $16 \mathrm{~h}$ were pre-treated with formononetin for indicated times. Following washing with fresh medium, the cells were stimulated with LPS for $24 \mathrm{~h}$. Equal volumes of conditioned media were analyzed by immunoblotting. Ponceau S staining was used as the loading controls. (E) Cells cultured in serum-free medium for $16 \mathrm{~h}$ were incubated with $\mathrm{CHX}$ or $\mathrm{Act} \mathrm{D}$ in the presence or absence of formononetin. After incubation for $24 \mathrm{~h}$, total RNA was isolated and the levels of SIRT1 mRNA were analyzed by real-time PCR. The results are expressed as the means $\pm S E(n=3)$. (F) Cells were transfected with siRNA against PPARס or control and grown for $38 \mathrm{~h}$. The cells were then lysed and aliquots of whole-cell lysates were subjected to Western blot analysis. (G) Cells transfected with PPARd-targeting or control siRNA for $38 \mathrm{~h}$ were stimulated with formononetin for $24 \mathrm{~h}$. Aliquots of whole-cell lysates were analyzed by immunoblotting. $(\mathrm{H})$ Cells transfected with $1 \mu \mathrm{g}$ of the SIRT1 luciferase reporter plasmid and $0.5 \mu \mathrm{g}$ of $\mathrm{pSV}$ b-Gal for $38 \mathrm{~h}$ were pretreated with GSK0660 for $30 \mathrm{~min}$ and then exposed to formononetin for $24 \mathrm{~h}$. Luciferase activity was normalized to $\beta$ galactosidase activity. The results are expressed as the means \pm SE $(n=3) .{ }^{*} p<0.05,{ }^{* *} p<0.01$ compared with the untreated group; ${ }^{*} p<0.05$ compared with the LPS-treated group; ${ }^{\dagger} p<0.05$ 
compared with the formononetin-treated group.

A

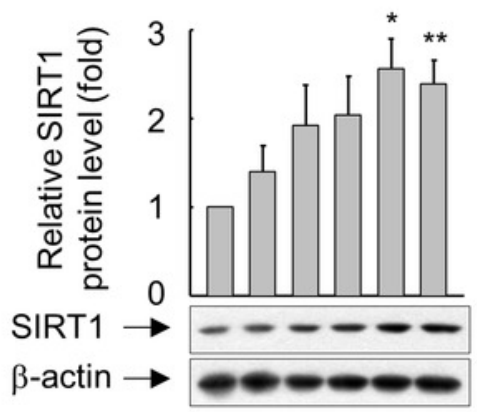

Formononetin $(\mu \mathrm{M}) \quad 0 \quad 15102030$

Time (24 h)
B

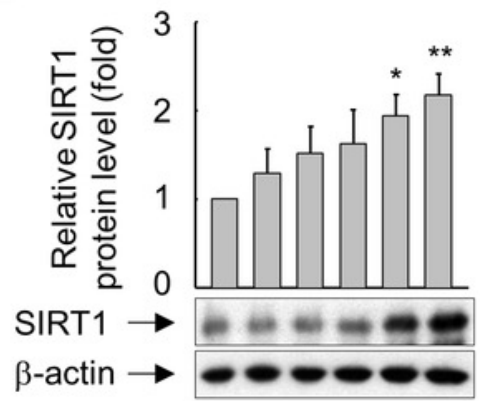

Time (h)
C

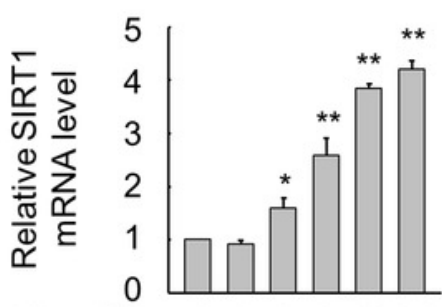

Time (h)

Formononetin $(30 \mu \mathrm{M})$

E

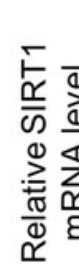

Formononetin $(30$ $\mathrm{CHX}(100 \mathrm{nM})$ Act $\mathrm{D}(100 \mathrm{nM})$

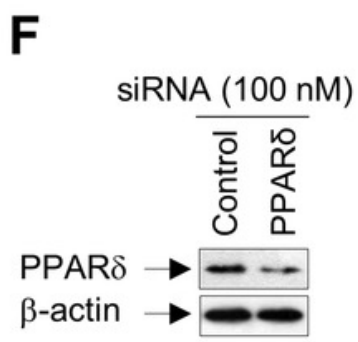

G

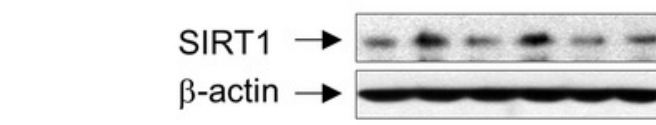

Formononetin $(30 \mu \mathrm{M})$ PPAR $\delta$ siRNA (100 nM) Control siRNA (100 nM)
H

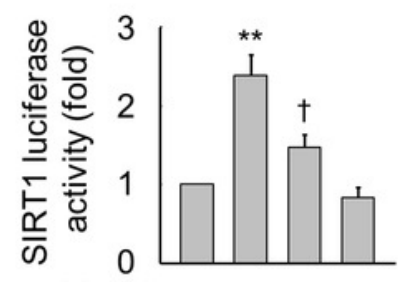

Formononetin $(30 \mu \mathrm{M})-++$ $\operatorname{GSK} 0660(10 \mu \mathrm{M}) \quad-\quad++$ 


\section{Figure 3}

Involvement of SIRT1 in the formononetin-mediated inhibition of LPS-induced HMGB1 release in RAW264.7 cells.

(A) Cells cultured in serum-free medium for $16 \mathrm{~h}$ were stimulated with LPS in the presence or absence of formononetin for $24 \mathrm{~h}$. (B) Cells were transfected with siRNA against SIRT1 or control and incubated for $38 \mathrm{~h}$. (C) Cells transfected with or without SIRT1-targeting siRNA for $38 \mathrm{~h}$ were exposed to LPS in the presence or absence of formononetin for $24 \mathrm{~h}$. (D and E) Cells pretreated with sirtinol (D) or resveratrol (E) for 30 min were stimulated with LPS in the presence or absence of formononetin for $24 \mathrm{~h}$. Equal volumes of conditioned media or aliquots of whole-cell lysates were analyzed by immunoblotting with the indicated antibodies. Ponceau S staining and $\beta$-actin were used as a loading control. Representative blots are provided. The fold changes in the SIRT1/ $\beta$-actin or HMGB1/Ponceau S ratio relative to that in the untreated group are shown as means \pm SE $(n=3) .{ }^{* *} p<0.01$ compared with the untreated group; ${ }^{\#} p<0.05,{ }^{\# \#} p<0.01$ compared with the LPS-treated group; ${ }^{\dagger} p<0.05$ compared with the LPS plus formononetin-treated group. 
A

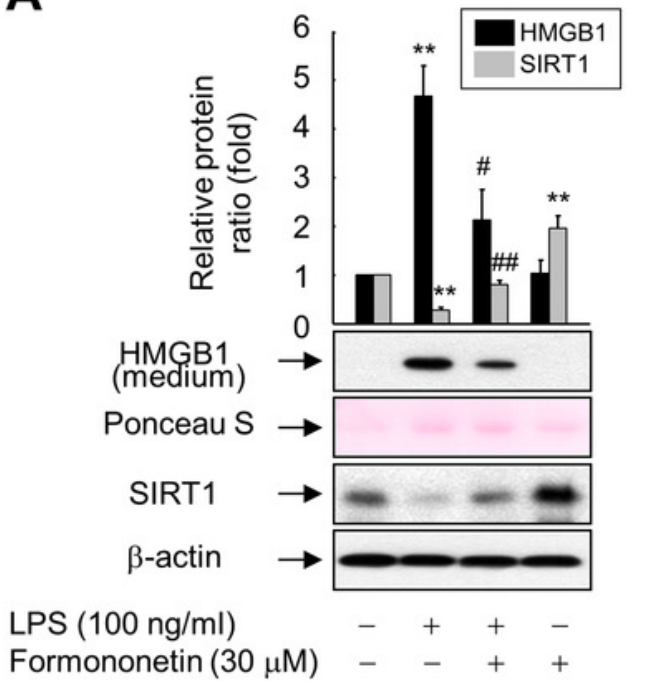

B

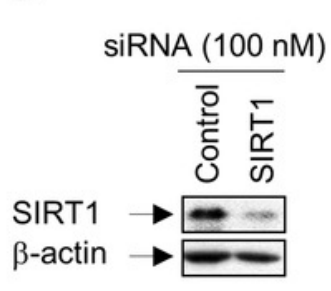

C

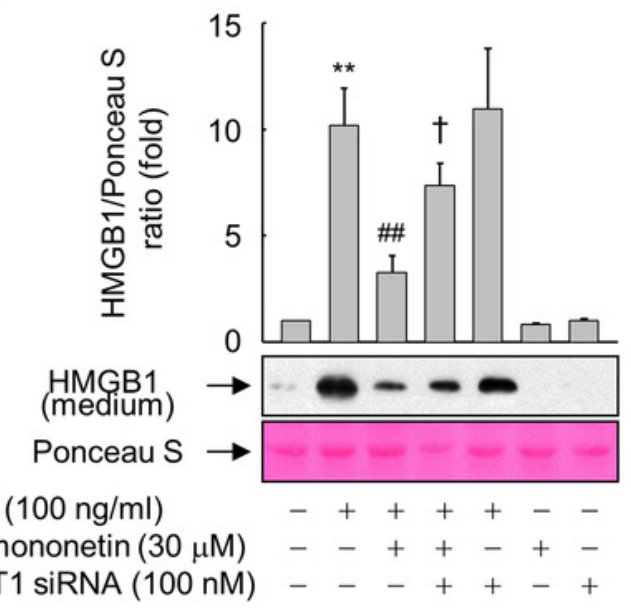

E

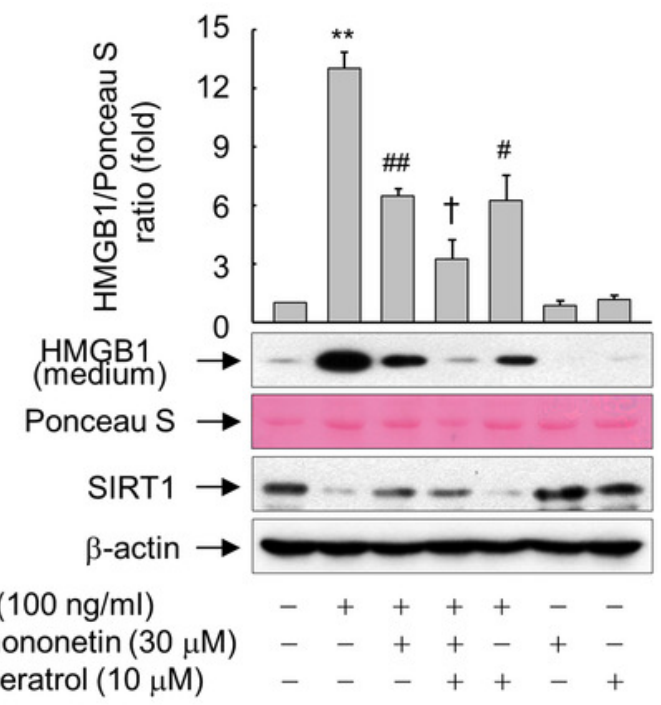




\section{Figure 4}

Effect of formononetin on LPS-induced HMGB1 acetylation.

(A) RAW264.7 cells cultured in serum-free medium for $16 \mathrm{~h}$ were stimulated with LPS in the presence of increasing concentrations $(10,20$, and $30 \mathrm{mM}$ ) of formononetin for $6 \mathrm{~h}$. (B and C) Cells transfected with SIRT1-targeting siRNA (B) or PPARס-targeting siRNA (C) for $38 \mathrm{~h}$ were exposed to LPS in the presence or absence of formononetin for $6 \mathrm{~h}$. Whole-cell lysates were immunoprecipitated with an anti-HMGB1 antibody, and then acetylated HMGB1 was detected by immunoblot analysis with an anti-acetyl-lysine antibody. Representative blots are provided. The fold changes in the acetylated HMGB1/total HMGB1 ratio relative to that in the untreated group are shown as means $\pm S E(n=3) .{ }^{* *} p<0.01$ compared with the untreated group; ${ }^{*} p<0.05,{ }^{\#} p<0.01$ compared with the LPS-treated group; ${ }^{\dagger} p<0.05$ compared with the LPS plus formononetin-treated group.

A

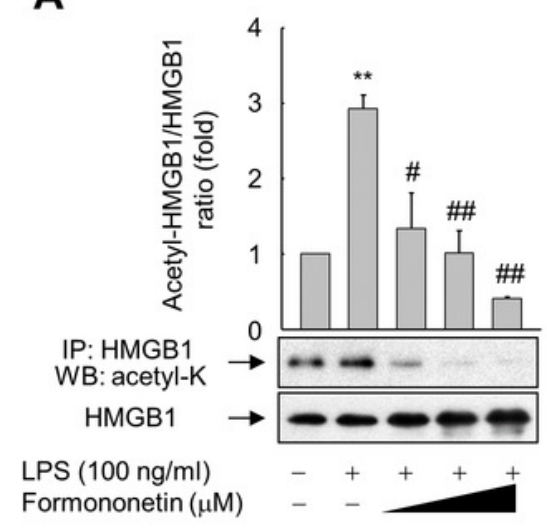

B

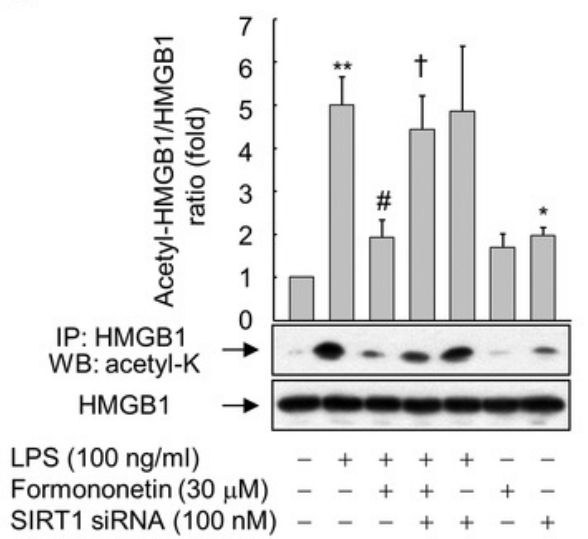

C

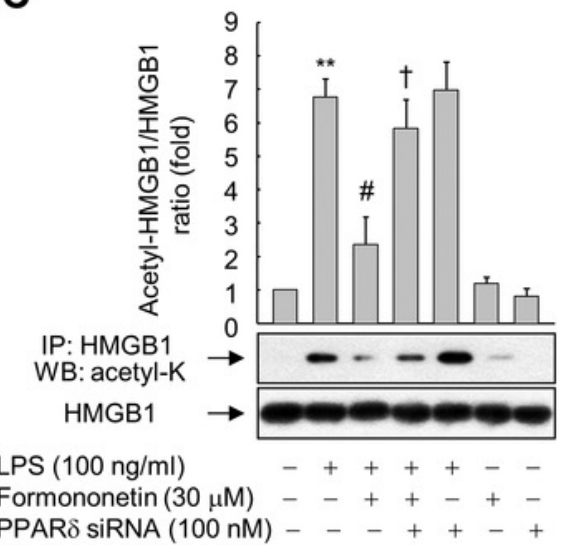

